\title{
USO DEL VIDEOBLOG PARA UN APRENDIZAJE COLABORATIVO DE SEGUNDAS LENGUAS CON ALUMNADO INMIGRANTE
}

\author{
THE USE OF VIDEOBLOGS AS COLLABORATIVE TOOLS FOR \\ INMIGRANTS STUDENTS WHEN LEARNING SECOND LANGUAGES
}

\author{
Urtza Garay Ruiz; urtza.garay@ehu.es \\ Carlos Castaño Garrido; carlos.castano@ehu.es \\ Universidad del País Vasco
}

\section{RESUMEN}

El objetivo de esta investigación es analizar los resultados de una intervención educativa dirigida a alumnado inmigrante basada en el uso de herramientas de la Web 2.0 para desarrollar un proceso de enseñanza-aprendizaje de segundas lenguas. Las técnicas de recogida y gestión de datos utilizadas fueron tanto cuantitativas como cualitativas. Uno de los resultados más relevantes es que los videoblogs son una herramienta que posibilita una enseñanza de segundas lenguas cooperativa y centrada en la comunicación. ${ }^{1}$

PALABRAS CLAVE: Vlog, aprendizaje colaborativo, aprendizaje de segundas lenguas, alumnado inmigrante.

\begin{abstract}
We present a study that analyzes the results of an educational intervention. The educational intervention was done with immigrant students and is based on the use of web 2.0) to develop a process of teaching and learning of second languages. We use qualitative and quantitative techniques to collect data. One of the most important results is that the videoblogs are a tool that enables active second language teaching, cooperative and focused on communication.
\end{abstract}

KEY WORDS: Vlog, cooperative learning, second language learning, immigrant students.

\footnotetext{
${ }^{1}$ Este artículo es el resultado del Proyecto de Investigación IT 448-10, "Contenidos digitales educativos", implementado con la financiación del Departamento de Educación, Universidades e Investigación del Gobierno Vasco en el período $2010-2016$.
} 


\section{INTRODUCCIÓN}

En los últimos años, continuamente estamos recibiendo en nuestras aulas alumnado procedente de diversos lugares de Europa y el mundo, hecho que supone estar preparado para darles la atención necesaria desde el punto de vista lingüístico, ya que cada vez conviven más lenguas en las aulas y el profesorado debe afrontar el reto de gestionarlas de forma eficaz.

Pero estos alumnos inmigrantes, pese a desconocer las dos lenguas oficiales autóctonas, no son analfabetos lingüísticos; conocen sus lenguas. No solo su lengua materna, sino hasta una, dos o incluso tres más. Por lo que tienen recursos lingüísticos y gramaticales; por ello, el proceso de enseñanza-aprendizaje de segundas lenguas no debe partir de cero (Huget, 2009; Chireac, 2011; Huguet \& Janes, 2013), sino desde el principio de que todas las lenguas sirven para "hacer cosas" (Besalú, 2008).

Por lo tanto, la enseñanza de segundas lenguas se debe comprender desde un punto de vista que compatibilice el enfoque constructivista, en el sentido de hacer cosas con las palabras, con el enfoque comunicativo, entendiendo las lenguas como elemento esencial para la comunicación Pero más allá de favorecer la unión entre lo constructivista con lo comunicativo, uno de los factores que más repercute a la hora de aprender una lengua es la voluntad de aprenderla. Es decir, desear introducirse en el mundo que envuelve dicha lengua. Este sentimiento, a pesar de que se vive de manera individual, supone una responsabilidad social. El deseo no parte únicamente del deseo individual, ni de la necesidad; sino también de los hablantes de la lengua que se va a aprender, la acogida lingüística y emocional que se recibe, y la edad con la que llega el alumno al centro de enseñanza (Vila, 1999). Es por ello que la clase de segundas lenguas debe convertirse en un lugar donde caben todas las lenguas, y todas ellas tienen relevancia, ya que ésta es la forma de poder reconocer lo que el alumnado inmigrante aporta a nuestras aulas: sus lenguas, y como afirman diferentes estudios (Bernaus, Moore \& Cordeiro, 2007; Huguet, Janes \& Chireac, 2008; Níkleva, 2009; Lapresta, Huguet \& Janés, 2010; Garay, 2011) los alumnos inmigrantes que se sienten más valorados desarrollan actitudes más favorables hacia las lenguas que están aprendiendo, aspecto que revierte sobre sus aprendizajes lingüísticos (Huguet y Janés, 2013).

Así, una de las formas más naturales para la gestión del uso de todas las lenguas de los alumnos en clase es por medio de la comunicación real que implica de forma directa la colaboración; esto es, la enseñanza colaborativa garantiza que todo el alumnado encuentre su lugar para expresarse y presentar su contribución a los demás. Pues mediante el aprendizaje colaborativo se comparte la autoridad y, entre todos, se acepta la responsabilidad de las acciones del grupo (Sotomayor, 2010).

Proponemos en esta investigación la utilización del videoblog o blog audiovisual (vlog) como instrumento para gestionar el proceso de enseñanza aprendizaje de lenguas basado en la colaboración. Diversos estudios indican que los blog son un instrumento adecuado para la interacción en diversos contextos interculturales (Coscollola \& Agustó, 2010; Larrea, Raigón \& Gómez, 2012). En el caso de la intervención que presentamos consideramos que los vlogs son un instrumento eficaz para introducir las lenguas propias del alumnado inmigrante en el aula y para que estos alumnos se 
conviertan en protagonistas y primeros responsables de su proceso de aprendizaje de segundas lenguas (Zhang \& Zhao, 2011; Bustamante, Hurbult \& Moelles, 2012; Chartrand, 2012). Por lo tanto, además de "hacer cosas" con sus lenguas de origen y con las lenguas que están aprendiendo deberán comunicarse y crear redes de aprendizaje entre iguales tanto en la Red (Siemens, 2007) como en el aula, lo que contribuirá a mejorar su propia autoestima lingüística y personal, algo que consideramos podrá aumentar el deseo por aprender las lenguas de acogida.

\section{CONTEXTUALIZACIÓN DE LA INVESTIGACIÓN}

La investigación se desarrolla en la Comunidad Autónoma del País Vasco CAV), por lo que el alumnado participante será acogido en las dos lenguas oficiales de la CAV (euskera y castellano) y su característica principal es la edad; es decir, son adolescentes. La adolescencia es una etapa de la vida en la que se desarrolla la identidad, y ese desarrollo supone la construcción del "yo" a través de los demás. Por lo cual los demás y sus lenguas son indispensables en la construcción de la identidad y la personalidad, porque representan un ejemplo a seguir (Funes, 1999; Giró Miranda, 2007; Garay, 2011).

Lo complicado es encontrar los medios para canalizar y difundir esas aportaciones lingüísticas que realizan los alumnos inmigrantes. Esto es, la manera en la que estos alumnos puedan sentir que sus aportaciones son tan necesarias como las de sus compañeros, y que perciban que las lenguas que forman parte de su identidad son tan importantes como las lenguas del centro de enseñanza, y que tanto unas como otras son útiles y beneficiosas para la construcción de su personalidad. Es aquí donde Internet cobra un papel importante, tal y como ya hemos comenzado a describir en el apartado anterior.

La Red, especialmente la Web 2.0, debido a su naturaleza, ofrece vías para trabajar la lengua de un modo comunicativo (utilizando la lengua para comunicarse) y constructivista (construyendo cosas a través de la lengua). Más allá, al hilo de lo planteado por Siemens (2007), nos adentramos en el conectivismo (Vela, 2012; Venkatachallam, 2013), complementando las teorías de aprendizaje antes citadas, y donde para aprender, incluyendo el aprendizaje de la lengua, es indispensable crear redes y alimentar las uniones; ya que es lo que nos impulsa a seguir aprendiendo a lo largo de la vida. Esto es, mediante Internet podemos hacer un aprendizaje colaborativo, donde aportamos y recibimos, donde escuchamos y nos escuchan, en definitiva, donde compartimos nuestras las lenguas para aprender otras nuevas.

Por ello en nuestra sociedad se define por lo audiovisual, esto es, vivimos en un entorno donde lo audiovisual cada vez se hace más presente en la vida cotidiana, y unido a ello, la publicación en tiempo real en Internet supone uno de los fenómenos más novedosos y extendidos del momento. Castaño, Maíz, Palacio y Villarroel (2008) afirman que Youtube es el fenómeno de las generaciones jóvenes y adolescentes de casi cualquier rincón del mundo. Ese dato debe impulsar un cambio en el modo de entender y de proceder en la enseñanza en general y en la enseñanza de segundas lenguas en particular. 
Lo audiovisual se ha naturalizado y goza de un protagonismo en la vida cotidiana y en la comunicación de los adolescentes, y sus componentes principales son tanto la imagen como el contenido. Por lo que una cápsula audiovisual resulta más representativa que mil palabras estáticas. Si la imagen es breve se convierte en microcontenido, donde además de la imagen pueden entrar en juego el sonido y la escritura, y convertirse en una herramienta atrayente al alcance de los agentes implicados en el proceso de enseñanza-aprendizaje de segundas lenguas. Además, si lo insertamos en un vlog, torna en un recurso excelente que abre las puertas a la comunicación y la colaboración, ya que éstas son las características básicas de los blogs, sean o no audiovisuales. Así, y siguiendo las palabras de Dolores Dreig los videovlog serían, así, un elemento que reforzaría el sentimiento de comunidad, de cooperación y de aprendizaje (Dreig, 2007), ya que promueven la participación desde un sentimiento dual del individuo y comunidad (Amorós, 2007; Amorós, 2009; Bohórquez, 2008).

\section{OBJETIVOS E HIPÓTESIS}

El objetivo de esta investigación es demostrar la viabilidad y eficacia del uso de los videovlogs para una enseñanza-aprendizaje de la lengua oral basada en la cooperación. De este modo, la investigación se basa en tres hipótesis que se plantean a priori y que son las siguientes:

1.-El uso de metodologías activas basadas en herramientas Web 2.0 fomenta la actitud positiva ante el aprendizaje de la lengua.

2.-La web 2.0 fomenta el aprendizaje colaborativo de lenguas. 3.-El uso de instrumentos de la web 2.0 (los videoblogs) posibilita la presencia y utilización de todas las lenguas del aula de forma eficaz.

Con el objetivo de verificar o desechar las hipótesis, planificamos y realizamos una intervención en dos centros educativos de Educación Secundaria de Bizkaia. Una intervención en la que el alumnado inmigrante desarrolló la habilidad oral y escrita en castellano y/o euskera, mediante una práctica basada en el aprendizaje colaborativo.

La intervención educativa consistía en crear un videovlog en cada centro. Mediante estos vlogs se pretendía que el alumnado que llevaba más tiempo en el centro de enseñanza preparara un material real para aquellos que llegarán en el futuro. Es decir, estos vlogs ayudarán al alumnado inmigrante futuro a conocer las estructuras lingüísticas básicas que necesitará los primeros días de clase. Para crear los videovlog los alumnos debían basarse en su experiencia vital, en su proceso migratorio, en lo que sintieron y necesitaron los primeros días de clase y en lo que pensaban que podrían necesitar otros compañeros al llegar a un centro de enseñanza de un nuevo país con dos lenguas oficiales.

La tarea se les planteó a los alumnos participantes mediante una miniwebquest donde se les guiaba para reflexionar sobre las necesidades lingüísticas que tuvieron en sus primeros días de clase, y basándose en ellas crear unos pequeños micro-vídeos con subtítulos, donde simultáneamente aparecían los dos idiomas castellano-euskera y su lengua familiar. 


\section{MÉTODO}

\subsection{Participantes}

El grupo de participantes lo componían 30 alumnos de origen extranjero, con edades comprendidas entre 12 y 17 años, de dos centros públicos de Enseñanza Secundaria ubicados en el Duranguesado, Bizkaia. Todos ellos llevaban menos de dos años en el sistema educativo español y provenían de Marruecos, Rumanía y Rusia. Además, se entrevistó a dos expertos responsables del Centro de Profesorado de Durango dependiente del Gobierno Vasco (Berritzegune de Durango) y se pasaron cuestionarios a 10 profesores y dos PRLs (Personal de Refuerzo Lingüistico) que trabajaban con el alumnado participante en la intervención educativa.

\subsection{Procedimiento}

Desde el punto de vista metodológico hemos llevado a cabo una investigación mixta, ya que se ha empleado la misma muestra para hacer diferentes análisis. Así, la investigación cuenta con un análisis cuantitativo y otro cualitativo. Podemos afirmar que la realización de investigaciones que comprenden ambos enfoques es una tendencia creciente. Los expertos (Cabero, 2004; Llorente, 2004) aseguran que es el procedimiento más adecuado para investigar la relación entre TIC y la educación. En nuestro caso incorporamos el factor aprendizaje de lenguas a la relación citada.

El objetivo principal es complementar las dos metodologías, siempre considerando las ventajas y desventajas que ambas presentan. Pero la característica principal de esta investigación, además de la multi-investigación o la investigación mixta, es que a nivel didáctico, la experimentación dentro la propia aula es relevante, ya que con este trabajo se pretende presentar y analizar lo que se hace en las aulas y crear una experiencia válida para el futuro.

Lo referente a la recopilación de datos se realizó mediante diferentes instrumentos que son los siguientes:

a. La recopilación de información y opiniones del alumnado participante se realizó mediante encuestas antes y después de la intervención. El diseño de las encuestas se basaba en el planteado por Osgood (1976).

b. El punto de vista del profesorado en general y del profesorado encargado del apoyo lingüístico al alumnado inmigrante se realizó mediante un cuestionario previo a la intervención educativa.

d. La opinión de los técnicos del Centro de Profesorado se recopiló mediante entrevistas antes y después de la intervención.

e. El investigador escribía un diario donde anotaba todos los detalles unidos a la investigación, centrándose sobre todo en la intervención educativa realizada en los dos centros de enseñanza secundaria. 
En lo que se refiere al propio análisis de los datos, para el análisis cuantitativo se recurrió al programa SPSS 11.5 mediante el cual se gestionaron los datos con un análisis descriptivo cimentado en frecuencias, porcentajes, desviaciones típicas y significatividad basada en la ANOVA. Para este análisis se obtuvieron los datos de los cuestionarios pasados al alumnado antes y después de la intervención educativa.

Para analizar los datos cualitativos se utilizó Atlas.ti 5.0 donde se gestionaron los datos obtenidos de las entrevistas y el diario del investigador. Para dicho análisis se creó una unidad hermenéutica donde se analizaron los datos cualitativos mediante la creación de códigos, MEMOS, familias y super-familias. Del que se obtuvieron resultados de dos tipos: textuales y de relación entre variables resumidas en códigos.

\section{RESULTADOS}

Los resultados cuantitativos se presentan conjuntamente con los cualitativos para cada una de las tres hipótesis de la investigación, y por último se relacionan las hipótesis entre sí.

La razón de presentar los resultados de manera conjunta reside en que en las tres hipótesis planteadas toma especial relevancia el uso de las herramientas que la Web 2.0 ofrece (miniwebquest, y videoblogs) y que son el eje de esta investigación.

A este respecto, antes de presentar los resultados en función de cada una de las hipótesis conviene señalar que el uso de Internet era habitual entre los alumnos; un $70 \%$ de los encuestados declaraba usarlo. Y la mayoría, un 51\%, lo utilizaba, sobre todo, para hablar con los amigos, esto es, para comunicarse. En consecuencia, siguiendo las pautas del enfoque comunicativo, resulta lógico que una herramienta destinada a la comunicación sea empleada en la enseñanza y el aprendizaje de lenguas.

Pero si analizamos los porcentajes que corresponden a la proporción en la que se usa Internet, según los alumnos participantes, en las clases de segundas lenguas (tanto en euskera como en castellano) queda de manifiesto que las herramientas de la web se usan poco:

\begin{tabular}{|c|c|c|c|c|c|}
\hline \multicolumn{6}{|c|}{ Utilizo Internet en las clases de español } \\
\hline & & Frecuencia & Porcentaje & $\begin{array}{c}\text { Porcentaje } \\
\text { válido }\end{array}$ & $\begin{array}{l}\text { Porcentaje } \\
\text { acumulado }\end{array}$ \\
\hline \multirow[t]{3}{*}{ Válido } & Poco & 25 & 73,7 & 73,7 & 73,3 \\
\hline & $\begin{array}{l}\text { Much } \\
\text { o }\end{array}$ & 5 & 26,3 & 26,3 & 100,0 \\
\hline & Total & 30 & 100,0 & 100,0 & \\
\hline
\end{tabular}

Tabla 1: Respuestas al ítem “Utilizo Internet en las clases de español”, SPSS 11.5. 


\begin{tabular}{|c|c|c|c|c|c|}
\hline \multicolumn{6}{|c|}{ Utilizo Internet en las clases de euskera } \\
\hline & & Frecuencia & Porcentaje & $\begin{array}{c}\text { Porcentaje } \\
\text { válido }\end{array}$ & $\begin{array}{l}\text { Porcentaje } \\
\text { acumulado }\end{array}$ \\
\hline \multirow[t]{4}{*}{ Válido } & Poco & 10 & 90,9 & 90,9 & 90,9 \\
\hline & Much & 1 & 9,1 & 9,1 & 100,0 \\
\hline & 0 & & & & \\
\hline & Total & 30 & 100,0 & 100,0 & \\
\hline
\end{tabular}

Tabla 2: Respuestas al ítem "Utilizo Internet en las clases de euskera", SPSS 11.5.

Así aunque el uso real es escaso el alumnado participante en la intervención expresaba que sí le gustaría utilizar Internet para aprender lenguas, tal y como se puede observar en las respuestas al item "Me gustaría utilizar Internet para aprender lenguas en el instituto", donde un $85 \%$ respondió que "mucho" y sólo un $15 \%$ que "poco".

Hipótesis 1: "El uso de metodologías activas basadas en herramientas Web 2.0 fomenta la actitud positiva ante el aprendizaje de la lengua oral"

Tras utilizar herramientas de la web para realizar las actividades planteadas en la intervención educativa, las percepciones de los participantes fueron positivas. Así, podemos señalar que al $85 \%$ le gustó mucho ver su trabajo colgado en Internet; al $83,3 \%$ le gustó mucho que el resto de compañeros y grupos pudieran ver el trabajo de su grupo en Internet; al $80 \%$ le gusto mucho que los profesores pudieran acceder a su trabajo mediante la web; y lo que es aún más importante, el 83,3\% tiene intención de seguir usando Internet para aprender lenguas, mediante el uso de los videovlogs grupales.

Los datos recopilados y analizados revelan que la utilización de la web como vía principal para trabajar aquello que envuelve la práctica oral de segundas lenguas ha sido valorado positivamente por los alumnos participantes, afirmando que han aprendido a hablar y manifestando su deseo de seguir introduciendo vídeos en el videovlog.

Así, después de la intervención, el 81,8\% manifestó que había practicado especialmente la lengua oral de la segunda lengua que estaba aprendiendo, tal y como se puede comprobar también en las siguiente citas extraídas por medio del programa de análisis de datos cualitativos Atlas.ti.

\section{P 6: DIARIO DE INTERVENCIÓN DE ZORNOTZA.txt - 6:33 [Alexandru habla despacio..] (165:165) (Super)}

Codes: [H6 ORALIDAD] [ALUMNADO]

Alexandru: Hemos aprendido a hablar despacio, con un tono alto.

Comment:

H6 
ZORNOTZA-INTERVENCIÓN-ALUMNADO

La pregunta es la siguiente: ¿Qué habéis aprendido?

P 6: DIARIO DE INTERVENCIÓN DE ZORNOTZA.txt - 6:28 [Alexandra le ha dicho dos veces..] (115:116) (Super)

Codes: [H6 ORALIDAD] [ALUMNADO]

Alexandra le ha dicho dos veces que vocalice más despacio que no se entiende si no.

El ambiente ha sido calmado, al principio sentían vergüenza... pero después han grabado los vídeos con naturalidad. Han tenido ocasión de reír, pero se lo han tomado en serio repitiéndolo tanto como les resultara necesario.

\section{P 6: DIARIO DE INTERVENCIÓN DE ZORNOTZA.txt - 6:29 [Les parece muy importante..] (116:116) (Super)}

\section{Codes: [H6 ORALIDAD] [ALUMNADO]}

Les ha parecido muy importante vocalizar despacio y alto, así los/las recién incorporados/as entenderían mejor.

Hipótesis 2: "La web 2.0 fomenta el aprendizaje colaborativo de las lenguas"

La cooperación nace de la misma filosofía del empleo de herramientas web 2.0. De todas formas, el enfoque colaborativo también se manifiesta en la planificación y desarrollo de las actividades de la intervención, donde continuamente se promueve el trabajo en grupo. Es decir, los alumnos hablan entre ellos de forma constante (negocian, se corrigen, etc.) en la segunda lengua y en sus lenguas maternas (entre aquellos que comparten la misma lengua o mediante la traducción). Además, valoran positivamente esta experiencia grupal, tanto por lo aprendido como por lo vivido. Para confirmarlo tenemos las siguientes citas:

\section{P 7: DIARIO DE INTERVENCIÓN DE IURRETA.txt - 7:6 [Se turnan para} grabar..] (44:44) (Super)

\section{Codes: [H5 COLABORATIVO] [ALUMNADO]}

Se han turnado para realizar la grabación, han decidió cómo y quiénes aparecerán y quién grabará. Por lo general lo han hecho entre dos, cada uno/a en un idioma, así quien lo ha hecho en español casi ha memorizado el texto. En otras ocasiones lo ha hecho la misma persona. Siempre con la ayuda del papel.

\section{P 6: DIARIO DE INTERVENCIÓN DE ZORNOTZA.txt - 6:12 [Alexandra habla en su idioma..] (112:112) (Super)}

Codes: [H2 VALORACIÓN] [H5 COLABORATIVO] [ALUMNADO] 
Alexandra lo hará en su idioma y con ayuda del papel, para recordarlo ya que el texto es demasiado largo. Además, ha comenzado con un "bueno" y Alexandru ha detenido la grabación: "bueno" no se dice en rumano.

P 6: DIARIO DE INTERVENCIÓN DE ZORNOTZA.txt - 6:24 [Te ha gustado trabajar con tus compañeros..] (142:143) (Super)

Codes: [H5 COLABORATIVO] [ALUMNADO]

¿Os ha gustado trabajar junto con vuestros/as compañeros/as? ¿Por qué?

Alexandra: Sí, me ha gustado porque así te dice lo que no sabes. Entre todos/as es más fácil.

Hipótesis 3: "El uso de instrumentos de la web 2.0 (los videoblogs) posibilita la presencia y utilización de todas las lenguas del aula de forma eficaz"

A pesar de que el $80 \%$ del alumnado pensaba que utilizar su lengua en el instituto sería positivo para su aprendizaje e integración y al $86,7 \%$ le gustaría aprender su lengua materna en el centro educativo y al 95,5\% poder utilizarla para aprender una segunda lengua, en los centros de enseñanza analizados no se utilizaba de forma sistemática la lengua de los alumnos inmigrantes, aunque los profesores veían la necesidad de utilizarlas.

\section{P 4: RESULTADOS ZORNOTZA.txt - 4:8 [Otras lenguas de los alumnos inmig..] (164:169) (Super)}

Codes: $\quad[\mathrm{H} 1][2 \mathrm{H}][$ PROFESORADO]

¿Se tienen en cuenta las lenguas de los alumnos inmigrantes en el instituto? ¿Cómo? ¿Para qué?
A. ...
B. No. Son cosas entre amigos. Alguna vez los portugueses intentaban aprender rumano. $C$.
D. Pocas veces. Traducir alguna canción a su lengua, o poner algún cartel hecho por ellos.

Después de la intervención el $84,4 \%$ valoró de forma positiva la presencia de sus lenguas dentro de la web para trabajar la lengua castellana, y el 90\% para trabajar la lengua vasca. La justificación de estos resultados la encontramos en el análisis cualitativo donde se aprecia que los alumnos inmigrantes se habían sentido valorados al poder demostrar que eran expertos en esas lenguas y porque habían podido aportar algo propio de sí mismos al instituto.
P 7: DIARIO DE INTERVENCIÓN DE ZORNOTZA 2.txt - 7:1 [Hemos
visto el blog..] (52:56) (Super) 
Codes: $\quad[\mathrm{H} 1+\mathrm{H} 2][$ ALUMNADO $]$

Hemos visto el vlog y hemos conversado sobre él a su nivel, teniendo en cuenta las dificultades de compresión, y sobre todo, expresión de este alumnado.

-Me gusta salir.

-Poner árabe es bonito.

-Aprendo castellano, pero con árabe.

-Divertido.

Por tanto, el empleo simultáneo de las lenguas les resulta satisfactorio a los alumnos participantes en la intervención. A pesar de que la actividad de subtitular los vídeos ha presentado dificultades, como metodología y sistema de aprendizaje ha sido valorado positivamente.

\section{P 6: DIARIO DE INTERVENCIÓN DE ZORNOTZA.txt - 6:23 [Alexandru ha dicho..] (129:129) (Super)}

\section{Codes: [H5 COLABORATIVO] [ALUMNADO]}

Alexandru ha dicho que cada vídeo debería tener un título para que sea fácil entender lo que se va a trabajar en cada vídeo, y así lo hemos hecho. En ocasiones en una lengua, en otras en otra, en general han empleado diferentes lenguas tal y como lo han hecho con los subtítulos.

\section{P 7: DIARIO DE INTERVENCIÓN DE IURRETA.txt - 7:3 [-Poner árabe es bonito] (54:54) (Super)}

Codes: [H2 VALORADA] [ALUMNADO]

-Poner árabe es bonito

Finalmente, estos resultados se refuerzan con el siguiente mapa conceptual que sustenta la relación que existe entre las tres hipótesis y el aprendizaje oral basado en la práctica: 


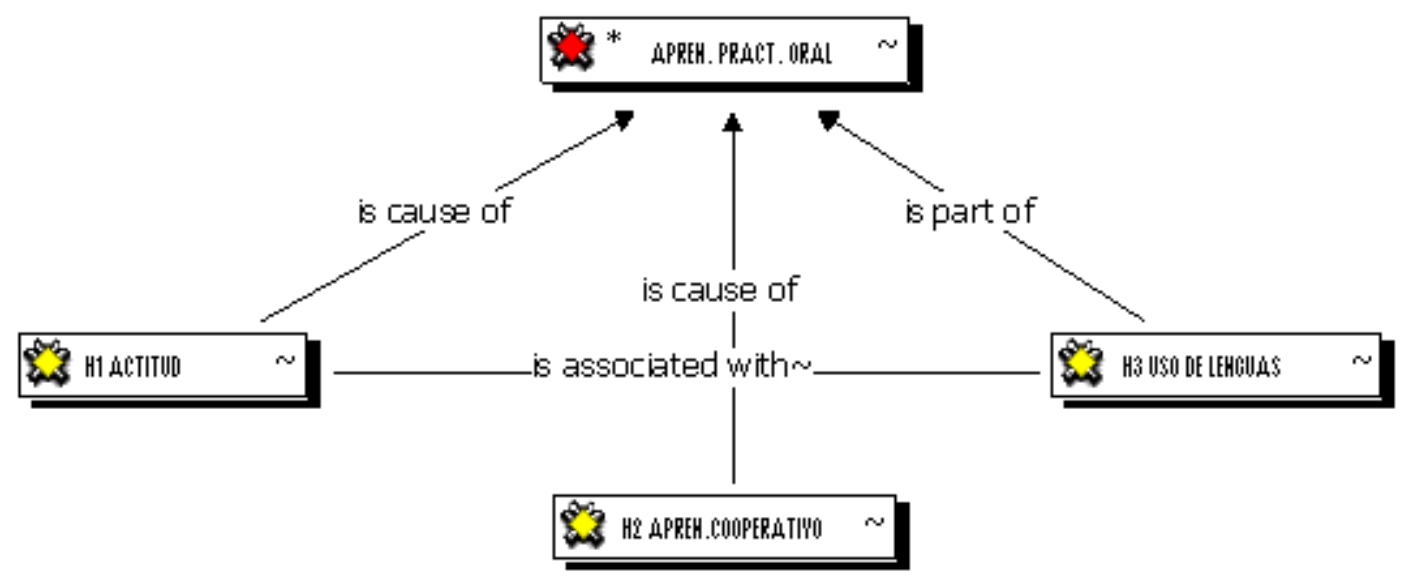

Figura 1: Relaciones jerárquicas entre variables. Atlas.ti 5.0

El esquema representa que los dos pilares básicos del aprendizaje de la segunda lengua, en este caso la práctica de la habilidad oral de la segunda lengua, son la actitud (Hipótesis 1) del alumno asociada con el uso de todas las lenguas (Hipótesis 2), que al mismo tiempo están asociadas con el aprendizaje colaborativo que queda en un segundo puesto, un poco por debajo de las otras dos hipótesis.

Pero, al mismo tiempo, se crean relaciones de causa entre el objetivo principal y las hipótesis uno y dos, esto es, la buena actitud $(\mathrm{H} 1)$ y el trabajo colaborativo $(\mathrm{H} 2)$ son la causa de ("is cause of") un buen aprendizaje práctico de la lengua oral, dentro del cual una parte ("is part of") importante la cubren la presencia y uso de todas las lenguas $(\mathrm{H} 3)$.

\section{DISCUSIÓN Y CONCLUSIONES}

Consideramos que la investigación evidencia de manera significativa que se puede llevar adelante una metodología cooperativa mediante las herramientas de la Web 2.0, y que dicha metodología cooperativa propicia que el alumnado se sienta partícipe directo del proceso su proceso de aprendizaje, pero también del proceso de enseñanza-aprendizaje de sus compañeros actuales y futuros. Ya que facilita el camino para que el alumnado se pueda relacionar con los elementos del centro escolar, como son el profesorado, el alumnado y el propio proceso de aprendizaje lingüístico.

Para ello es esencial encontrar caminos para que los alumnos extranjeros se sientan expertos, y no sólo aprendices de una segunda lengua, y, tal y como se ha podido comprobar, los vlogs se convierten en un instrumento que facilita la utilización de todas las lenguas en el proceso de enseñanza-aprendizaje de segundas lenguas. En consecuencia, la presencia de todas las lenguas posibilita que una parte importante de la identidad de estos alumnos inmigrantes sea compartida en el centro, haciendo que se sientan partícipes de la actividad escolar y potenciar su actitud positiva hacia las nuevas lenguas que deben aprender. Esto es, propician la comunicación entre iguales, 
lo que lleva implícito la cooperación como elemento esencial del aprendizaje y la práctica de las lenguas que se aprenden y se comparten.

Podemos concluir, por tanto, que el uso de los videoblogs posibilita una metodología fundamentada en el enfoque comunicativo y constructivista, pero que abre caminos al nuevo conectivismo, ya que proporciona facilidades para crear redes de relaciones que nos ayuden en el camino del aprendizaje lingüístico.

\section{BIBLIOGRAFÍA}

AMORÓS, L. (2007). Diseño de Weblogs en la enseñanza. Edutec. Revista Electrónica de Tecnología Educativa, 24, http://edutec.rediris.es/Revelec2/revelec24/lamoros/lamoros.htm, (22/04/2013).

AMORÓS, L. (2009). Weblogs para la enseñanza-aprendizaje, Píxel-Bit. Revista de Medios y Educación, 35, 61-71.

BERNAUS, M., MOORE, E. \& CORDEIRO, A. (2007). Affective factors influencing plurilingual students' acquisition of Catalan in a Catalan-Spanish bilingual context. The Modern Language Journal, 91(2), 235-246.

BESALÚ, X. (2008). ¿Cómo defenderse del alumnado extranjero? Cuadernos de Pedagogía. Barcelona: Praxis, 86-89.

BOHÓRQUEZ, E. (2008). El blog como recuso educativo. Edutec. Revista Electrónica de Tecnología http://edutec.rediris.es/Revelec2/revelec26/articulos n26 PDF/EdutecE Bohorquez n26-\%203.pdf, (23/04/2013).

BUSTAMANTE, C., HURLBUT, S. \& MOELLER, A. (2012). Web 2.0 and language learners: moving for consumers to creatores. En Sildus, T. (Ed.), Touch the world. Recuperado de http://www.csctfl.org/documents/2012Report.pdf\#page=89 (52-2013).

CASTAÑO, C.; MAÍZ, I.; PALACIO, G. \& VILLARROEL, J.D. (2008). Prácticas educativas en entornos web 2.0. Madrid: Síntesis.

COSCOLLOLA, M.D. \& AGUSTÓ, M.F. (2010). Innovación educativa: experimentar con las TIC y reflexionar sobre su uso. Pixel-Bit. Revista de Medios y Educación, 36, 171-180.

CHARTRAND, R. (2012). Social networking for language learners: creating meaningful output whit Web 2.0 tools, Knowledge Management and E-Learning: An International Journal, 1 (4), 97-101. Recuperado de http://www.kmeljournal.org/ojs/index.php/online-publication/article/viewFile/170/124 (7-22013). 
CHIREAC, S.M; SERRAT, E. \& HUGUet, Á. (2011). Procesos de transferencia en el aprendizaje de segundas lenguas. Un estudio con escolares de lengua rumana en un contexto educativo bilingüe. Revista de Psicodidáctica, 16(2), 267-289.

DREIG, D. (2007). “Actitudes, comportamientos, usos, clasificación de los usuariso de redes sociales" en http://www.dreig.eu/caparazon/2009/02/09/actitudescomportamiento-usos-clasificacion-de-los-usuarios-de-las-redes-sociales/, $(23 / 04 / 2013)$

FUNES, J. (1999). Migración y adolescencia. Recuperado de http://www.aulaintercultural.org/IMG/pdf/migracion_adolescencia (23-052013)

GARAY, U. (2011). Ahozkotasunaren ikaskuntza-irakaskuntza DBHko ikasle etorkinetan. Deskripzioa, esperimentazioa eta emitzen azterketa. (Tesis doctoral digitalizada). Universidad del País Vasco. Bilbao.

GIRÓ MIRANDA, J. (2007). Adolescencia, migración e identidad. En Joaquín Giró Miranda (coord.), La escuela del siglo XXI: la educación en un tiempo de cambio social acelerado: XII conferencia de sociología de la educación: Logroño, 14 y 15 de septiembre de 2006, 97-98.

HUGET, Á. (2009). La Hipótesis de Interdependencia Lingüística. Algunas consideraciones para la práctica educativa en aulas multilingües. Revista Española de Pedagogía, 244, 495-510.

HUGUET, Á., JANÉS, J. \& CHIREAC, S.-M. (2008). Mother tongue as a determin-ing variable in language attitudes. The case of immigrant Latin Ameri-can students in Spain. Language and Intercultural Communication, 8 (4), 246-260.

HUGUET, Á. \& JANES, J. (2013). Escuela e inmigración. Una presentación de estudios sobre el desarrollo de habilidades lingüísticas y procesos de interdependencia entre lenguas. Anales de Psicología, 29 (2), 393-403.

LAPRESTA, C., HUGUET, Á. \& JANÉS, C. (2010). Análisis discursivo de las actitudes lingüísticas de los escolares de origen inmigrante en Cataluña. Revista de Educación, 353, 521-547.

LARREA, A.M., RAIGÓN, A.R. \& GÓMEZ, M.E. (2012). ICT for intercultural competence development, Píxel-Bit. Revista de Medios y Educación, 40, 115-124.

NíKLEVA, D. G. (2009). La convivencia intercultural y su aplicación a la enseñanza de lenguas extranjeras. Ogigia: Revista Electrónica de Estudios Hispánicos, 5, 2940.

OSGOOD, C.E. (1976). La medida del significado. Madrid: Gredos.

SIEMMENS, G. (2007). Connectivism: Creating A Learning Ecology in Distributed Environments. Jug. T. (ed.) Didactics of Microlearning. Concepts, Discourses and Examples. Alemania: Waxmann. 
SOTOMAYOR, g. (2010). Las redes sociales como entornos de aprendizaje colaborativo mediado para segundas lenguas. Edutec. Revista Electrónica de Tecnología Educativa. 34, http://edutec.rediris.es/Revelec2/Revelec34/pdf/Edutece n34 Sotomayor.pdf, (20-05-2013).

VELA, A. (2012): Estilos de aprendizaje y teorías. Recuperado de http://alfredovela.files.wordpress.com/2012/08/estilosdeaprendizajeyteorias.j pg (22-05-2013)

VENKATACHALLAM, U (2013): Connectivism: learning theory for the future?. Recuperado de http://blog.appropriateit.org/2013/04/17/connectivismlearning-theory-for-the-future/ (22-05-2013)

VILA, I. (1999). Inmigración, educación y lengua propia. Recuperado de http://www.aulaintercultural.org/IMG/pdf/lenguapropia.pdf (22-11-2009).

ZHANG, G. \& ZHAO, Y. (2011). Technology uses in creating second language (L2) learning environments: When learners are creators. En Koehler, M. y Mishra, P. (Eds), Proceedings of Society for Information Technology and Teacher Education International Conference 2011. (pp. 2940-2945). Chesapeake: VA: AACE

\section{Para citar este artículo:}

GARAY, U. \& CASTAÑO, C. (2013). Uso del videoblog para un aprendizaje colaorativo de segundas lenguas con alumnado inmigrante. EDUTEC, Revista Electrónica de Tecnología Educativa, $46 . \quad$ Recuperado el dd/mm/aa de http://edutec.rediris.es/Revelec2/Revelec46/videoblog aprendizaje colaborativo segundas I enguas alumnado inmigrante.html 